\title{
L'agir au travail du professeur de fitness
}

Le lien social rythmé par le corps et la technique

The fitness instructor's action at work. Social bond through body and technique

\section{Lilian Pichot}

\section{OpenEdition}

\section{Journals}

Édition électronique

URL : https://journals.openedition.org/tc/8430

DOI : $10.4000 /$ tc. 8430

ISSN : 1952-420X

Éditeur

Éditions de l'EHESS

\section{Édition imprimée}

Date de publication : 1 décembre 2014

Pagination : 252-271

ISBN : 978-2-7351-2346-9

ISSN : 0248-6016

Référence électronique

Lilian Pichot, «L'agir au travail du professeur de fitness », Techniques \& Culture [En ligne], 62 | 2014, mis en ligne le 01 décembre 2017, consulté le 29 septembre 2022. URL : http://journals.openedition.org/ tc/8430 ; DOI : https://doi.org/10.4000/tc.8430 


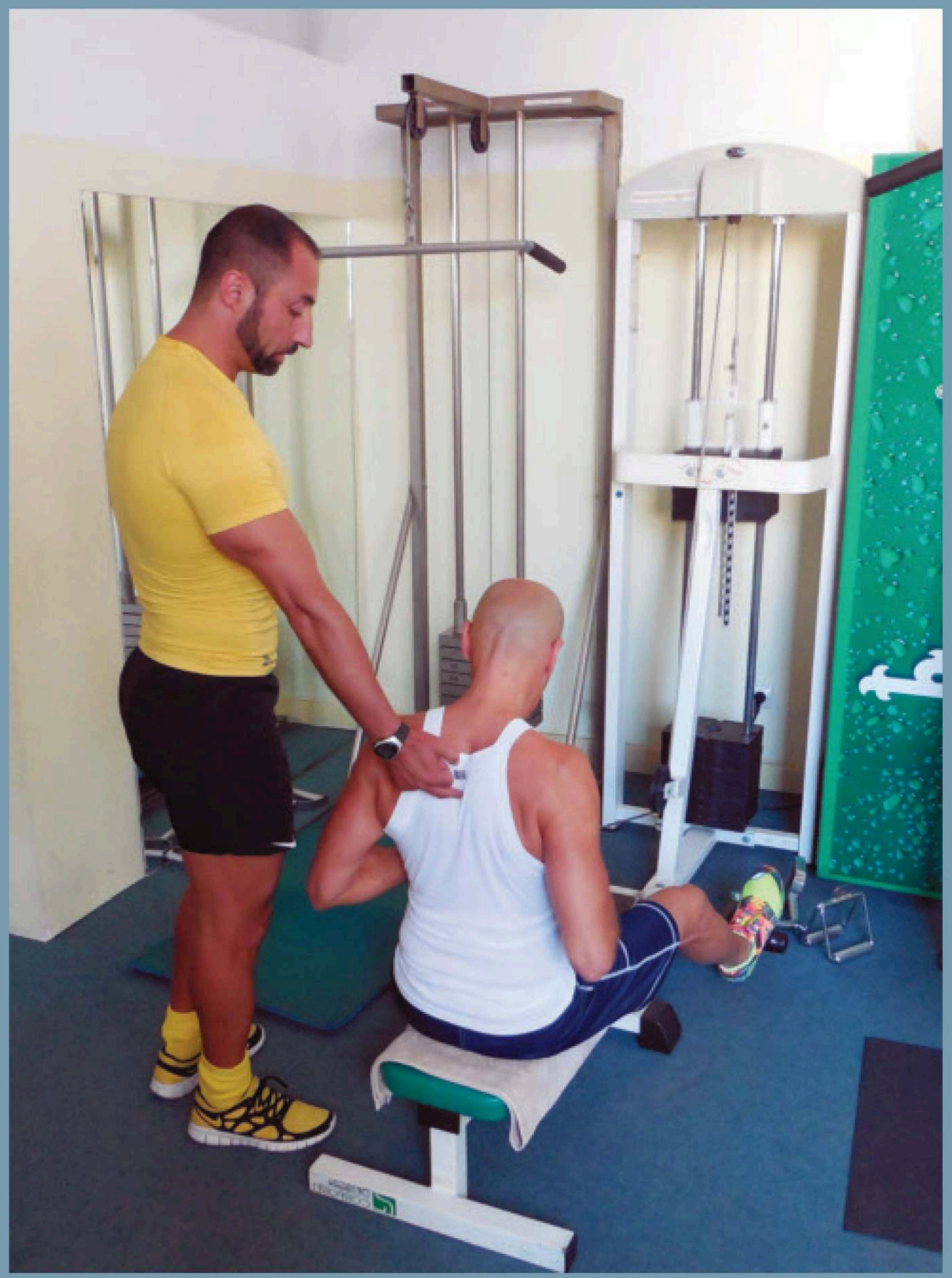




\section{L'AGIR AU TRAVAIL DU PROFESSEUR DE FITNESS}

\section{Le lien social rythmé par le corps et la technique}

Comment un travailleur tel que le professeur de fitness (PF) - éducateur sportif spécifique-s'accommode de l'hétérogénéité matérielle et humaine qui caractérise son lieu de travail? C'est à cette problématique que notre recherche tente de répondre ici, en regard d'observations qui ont permis de qualifier le monde social du fitness, marqué par des contrastes saisissants (Ernst \& Pigeassou 2005; Mischler \& Pichot 2005; Haissat \& Travaillot 2012). La variété des espaces de pratique dans les clubs de fitness traduit une différenciation sociale et technique des lieux. Selon les zones d'activités et d'expressions corporelles (salles de cours collectifs, zone de plateau de musculation), le rapport à autrui se définit dans des situations où règnent aussi bien l'impersonnalité des liens que la personnalisation des échanges. Ces configurations contrastées caractérisant l'espace de travail du PF l'engagent dans une activité composite, démultipliée. Comment a-t-il prise sur ce flux de personnes qui peuplent les salles de fitness et dont on ne connaît à l'avance que très partiellement les effectifs et les moments d'arrivée et de départ?

Le « vrai boulot» (Bidet 201la) oblige à saisir sur le vif les manières de vivre le travail, les corps qui le dirigent et les mots qui le parlent. L'examen de «l'activité en train de se faire» (Bidet et al. 2006), solidaire d'une approche écologique (Joseph 1998), s'applique à débusquer les ressources adaptatives pour s'ajuster continûment aux incertitudes qui rythment les phases successives au travail. Acquises et travaillées, ces ressources sont le fruit d'arrangements plus improvisés que planifiés, même s'ils sont maîtrisés, et reposent en grande partie sur des engagements sensibles, émotionnels et moraux (Pillon et al. 2011).

À partir d'une démarche méthodologique d'observation participante et non participante (Gold 1958) et, d'entretiens menés avec les professionnels du fitness, 
Plateau d'appareils de musculation et zone d’activité cardio-training composée de rameurs

et de tapis de course

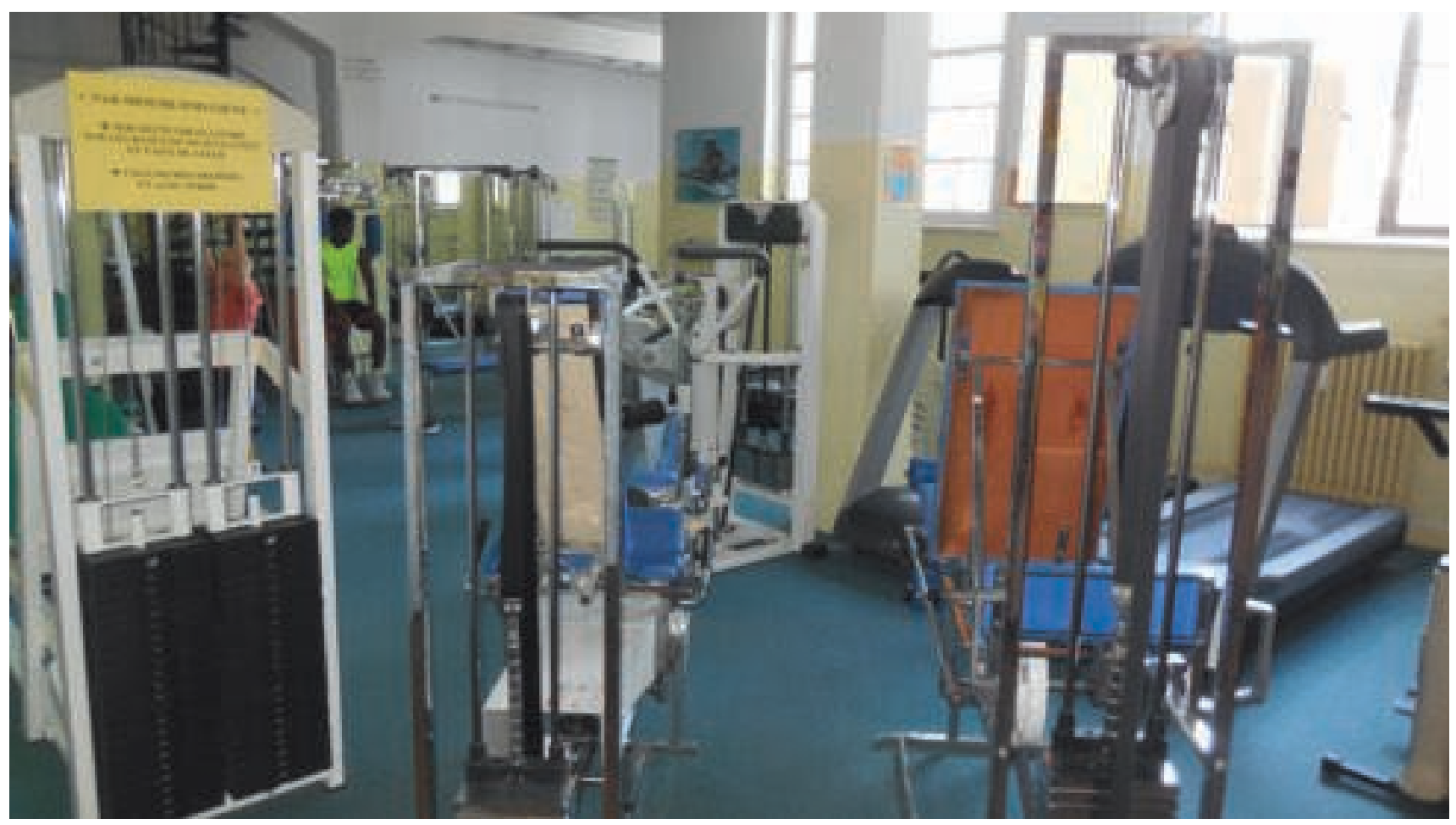

cette étude s'emploie à saisir la dynamique créatrice à laquelle participe le PF. En d'autres termes, il s'agit de mettre en exergue les manières dont l'instructeur use de son savoir-faire technique, de ses ressources physiques et corporelles, de ses savoir-être pour faire face aux obstacles du travail et aux tensions résultant d'attentes différentes. La formation de collectifs multiformes est ainsi constitutive de son identité professionnelle et de sa reconnaissance sociale. L'article tente de montrer que la rythmicité, définie comme une insertion affective et corporelle dans l'existence (Leroi-Gourhan 1965), est ancrée dans l'ajustement constant et maîtrisé du travailleur aux divers signaux d'action, et plus totalement au milieu dans lequel il agit. Et ce, alors même que les PF sont enjoints à gérer la dispersion, c'est-à-dire divers stimuli parfois contradictoires (Datchary 2008).

Comment, par ses manières d'être motrices et socialement construites, l'insertion affective dont il est question, est immanente à l'engagement au travail (Bidet 2011 a)? Comment est-elle signifiante aux yeux d'un ensemble humain fort complexe, éphémère et incertain? Quel est ce tissu de relations humaines et techniques qui fait sens pour les professeurs de fitness?

Lapproche transverse anthropologique du rythme (Leroi-Gourhan 1964, 1965) et l'ancrage pragmatiste concourent à décrypter les usages des expériences antérieures, d'une temporalité élargie qui donne à comprendre la fonction et le rôle joués par les expériences humaines vécues, professionnelles ou non, par les moments passés dans les coulisses (Goffman 1973), à l'arrière-plan de la scène du travail. 
Il nous importe alors d'appréhender l'hétérogénéité interne du travail (Bidet 2011 a, 2011 b) ou encore le travail protéiforme (Sennett 2000) qui définit l'univers des salles de fitness. Parce qu'«il [le travail humain] impose aussi aux travailleurs un "méta-travail" de gestion de leur disponibilité différenciée et de leur engagement : élaborer malgré tout des priorités, synchroniser ses activités, créer une continuité entre les épisodes qui se succèdent, les activités passées et présentes» (Bidet 2011b: 12).

En nous intéressant à l'exigence de cohérence et de continuité de l'expérience, loin de vouloir psychologiser l'engagement au travail, notre recherche aspire à saisir comment les formes sociales et les formes techniciennes sont articulées, et de quelles manières les expressions corporelles et humaines, propres à l'univers investigué, reposent sur une valeur technique et témoignent non moins de valeurs morales. Ce faisant, elles traduisent une solidarité technique (Dodier 1995) et corporelle au sein d'un ensemble humain hétérogène. Cette solidarité autour d'un collectif co-construit qui marque l'expérience de travail fait sens pour le PF qui y construit son identité.

\section{Le fonctionnement humain des ensembles techniques: quand les liens entre les êtres travaillent et redéfinissent la solidarité technique}

«Être seuls ensemble» (Ernst \& Pigeassou 2005: 65-74), cette expression traduit un paradoxe s'agissant d'un espace de travail d'un genre particulier, les clubs de fitness. Depuis plusieurs décennies, ces lieux apparaissent comme le chantre de l'individualisme contemporain, de l'exacerbation de soi pour ne pas dire de l'exhibition corporelle. Pourtant, ce sens commun élude trop rapidement les caractéristiques de situations de travail où règnent une organisation calquée sur des activités de consommations diverses et de fréquentes interactions humaines. Face aux adhérents-clients, l'action du salarié - en l'occurrence du PF - est intégrée à un ensemble composé de technologies et d'humains (Pichot \& Wipf 2011). Un des facteurs d'attractivité de ces salles, autrement appelées de (re) mise en forme, réside dans la dimension collective de la réponse apportée aux clients. Les services proposés sont multiples: cours collectifs d'intensité variable, avec ou sans l'utilisation de petits matériels de musculation, plus ou moins chorégraphiés; encadrement personnalisé dans la zone d'activation cardiaque et de training sur des machines adaptées (tapis roulant, vélo elliptique, rameur, etc.); espace d'activités de musculation en autonomie ou avec l'appui d'un conseiller et l'usage éventuel des appareils spécifiques. Seule une organisation temporelle et spatiale des ressources humaines permet d'assurer ces diverses prestations de services. Pour comprendre comment cet ensemble humain fait «corps», il convient de s'arrêter un instant sur les caractéristiques de l'espace de travail structuré par des médiations technologiques et humaines. L'observation de la structure spatiale des salles laisse apparaître un agencement réfléchi des différents espaces de pratique et des zones de transition spécifiques. Dans la plupart des cas, les salles de fitness se déploient sur plusieurs étages pour former une surface totale de pratique conséquente - allant le plus souvent de 500 à $1500 \mathrm{~m}^{2}$. Une zone d'une surface 


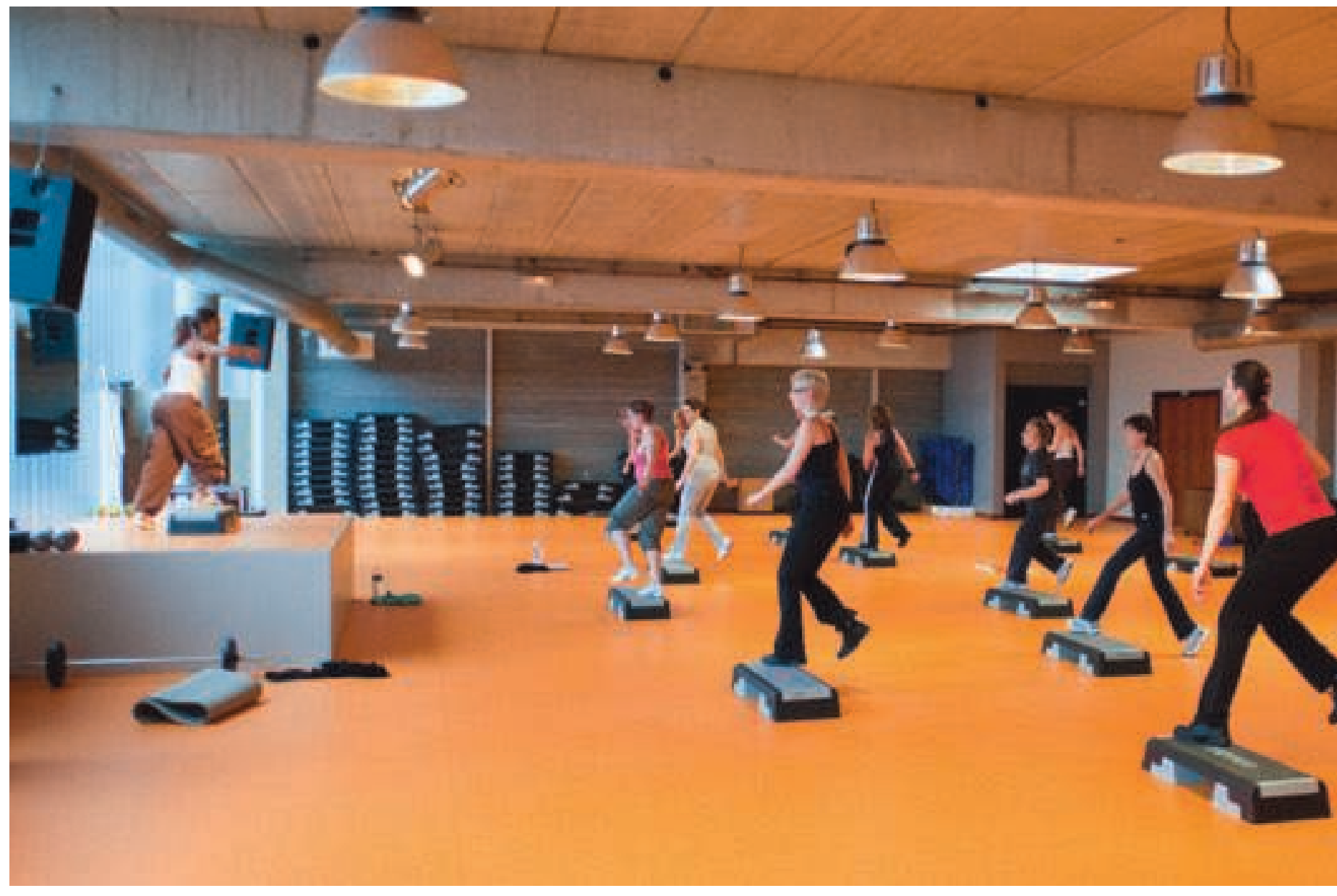

Exemples de salles de cours collectifs 


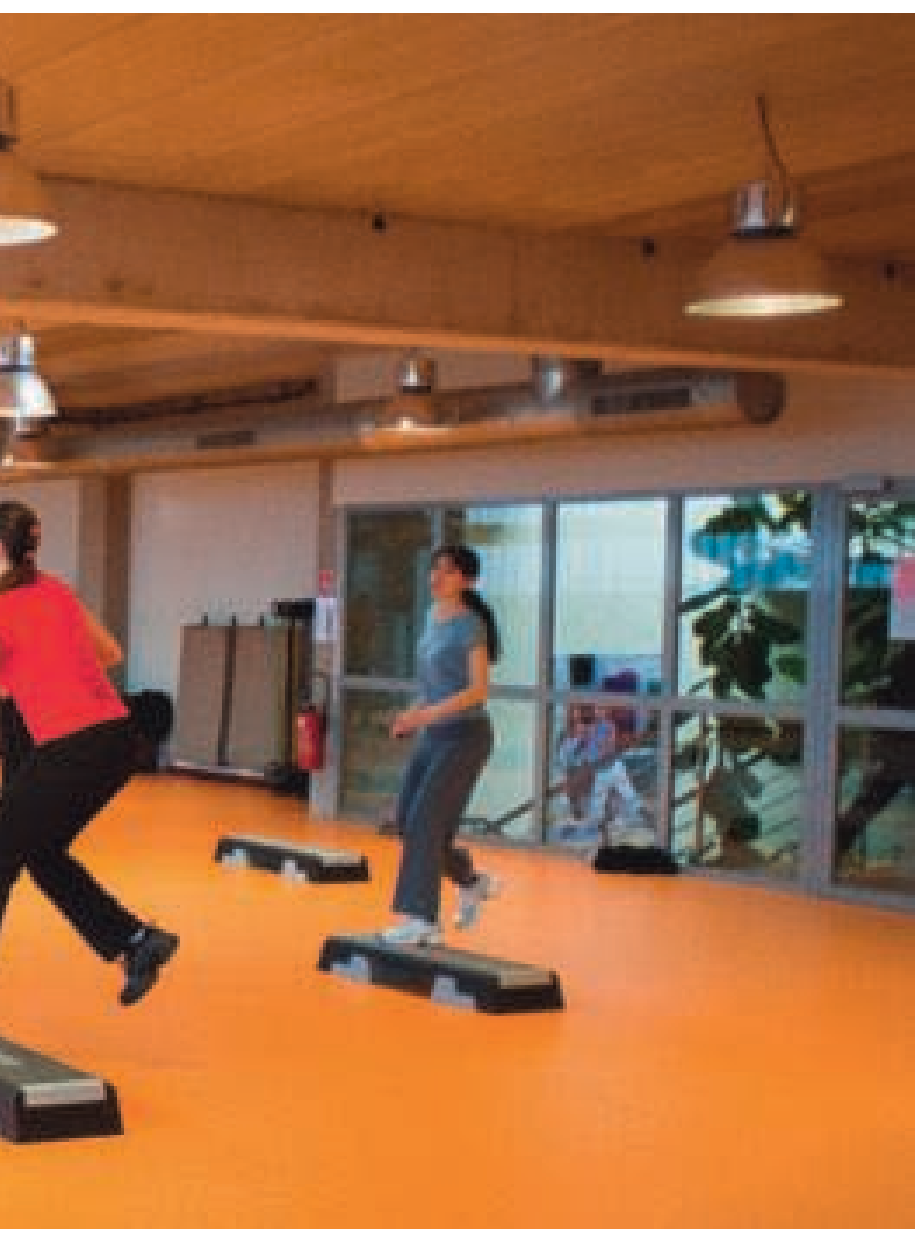

importante (150 à 200 m²) est dédiée au plateau de musculation équipé d'appareils guidés ou semi-guidés ainsi qu'à l'espace cardio-training. À un autre niveau, les salles de cours collectifs aux surfaces variables sont réparties, à partir d'un espace/anneau central, lieu de passage obligé des clients qui, en franchissant l'entrée du club, sont enregistrés et donc comptabilisés. Parfois, les salles de fitness sont équipées d'une piscine proposant les activités aquatiques (aquafitness, aguagym, aquabiking, etc.) essentiellement prisées par les femmes ${ }^{2}$ et dont le succès s'est établi grâce à leur caractère ludique et l'atténuation de l'épreuve physique. L'agencement spatial impacte les activités travaillées des PF et leur présence physique dans les différentes zones du club. En effet, la nécessité d'occuper en permanence les divers espaces de pratiques - « avoir toujours au moins deux professeurs dans l'entreprise pour répondre aux demandes des adhérents et les satisfaire »-participe de la solidarité technique, définie comme «une forme de liens entre les êtres créée par le fonctionnement des ensembles techniques 》 (Dodier 1995: 14). La co-activité fait partie du quotidien des PF qui tantôt assurent une prestation de service propre à leur domaine de prédilection dans des espaces spécifiques, et tantôt produisent une prestation sous la forme de binôme voire trinôme: «régulièrement en soirée au moment du pic d'affluence, on propose des cours en team teach, ça permet de créer un événement, un entraînement, une dynamique, de la cohésion entre nous», assure une PF, responsable des activités 


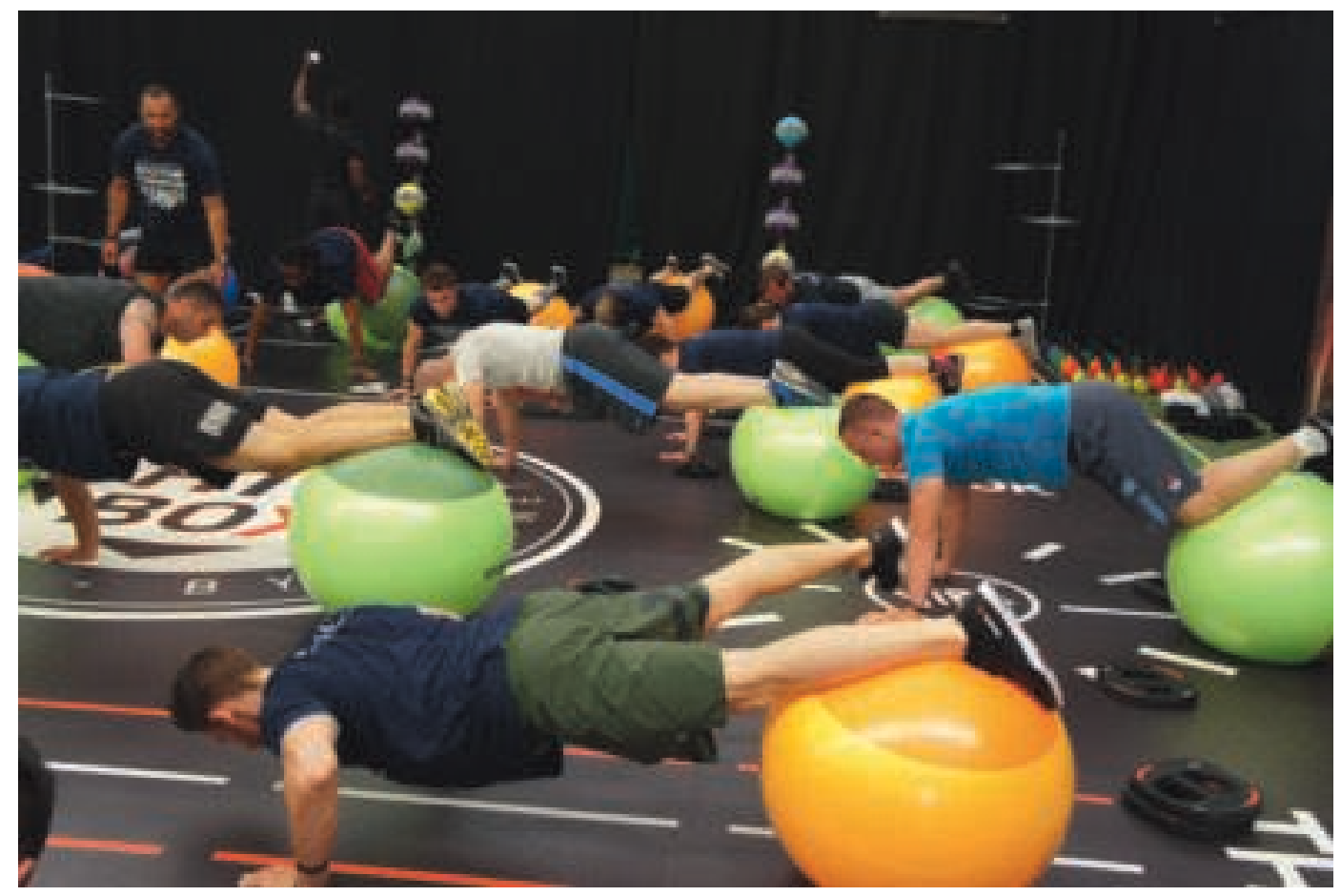

Exemples de salles de cours collectifs

travail - marqué par la différenciation et la complémentarité des zones géographiques du club - conduisent les professionnels à prévoir un travail collectif de répartition des tâches, dans le temps et dans l'espace, qui les rend interdépendants les uns des autres. L'instauration récente des circuits training ${ }^{3}$ fréquentés par des small group impose tacitement un respect individuel réciproque et collectif envers les déplacements des pratiquants sur les ateliers successifs, et ce sous l'œil avisé du PF. L'espace de cardio-training favorise la coopération entre pratiquants d'une part et, entre les clients et le professeur de fitness d'autre part. Outre le placement en vis-à-vis des appareils (tapis de course, vélos elliptiques) encourageant la communication interindividuelle, l'usage des machines prédispose à l'échange nécessaire à leur bonne utilisation. Co-activité, coprésence et présences répétées sont consubstantielles d'un espace équipé; il forge cette conscience collective liant durablement les individus entre eux (Dodier 1995: 48-494) et les amène à composer avec certaines exigences d'autrui. Les microcollectifs formés continûment, indexés aux agencements compartimentés de l'espace, sont défaits et reconstitués au gré des circonstances et des flux de fréquentation de la salle. Ici, deux ou trois personnes installées sur les machines de renforcement musculaire attirent le PF, il est convié à prodiguer quelques conseils de posture pour sécuriser les mouvements corporels; là, un groupe d'une dizaine de pratiquants se réunit pour suivre un cours de stretching dans une petite salle de $50 \mathrm{~m}^{2}$ prévue à cet effet. Le lieu intimiste encourage des attentions et des écoutes réciproques, ainsi que des gestes physiques placés, souples et exécutés sans retenue. La fonctionnalité de l'espace composé d'objets techniques exige une coordination minimale entre les salariés, pour le moins la transmission de consignes, de bilans de séances et de cours afin de suivre pas à pas l'évolution des pratiquants. 


\section{Le travail cadencé et rythmé par la co-activité du triptyque salarié-client-patron}

Pour comprendre ce qui fait sens dans cet univers du loisir sportif, il faut considérer l'engagement corporel, en particulier du PF, comme témoin d'une intentionnalité - au final satisfaire la clientèle - et comme pilote - générateur de pratiques et de comportements mimétiques. Mais loin de substantialiser l'investissement corporel, le point de vue relationnel donne à voir comment l'agir corporel du PF résulte tant du cadre normatif défini par l'employeur ou du manager que des pressions explicites ou latentes en provenance des clients en quête d'attentes et d'attentions particulières, sportives ou corporelles: «Moi je suis les cours de cuisses-abdos-fessiers pour tonifier tout ça, ben voilà quoi, la trentaine passée, un enfant [...] donc le prof, j'attends de lui qu'il nous propose un cours avec des exos accessibles qui vont me permettre de me renforcer musculairement» (K., 35 ans). À propos d'une PF récemment recrutée, un gérant de club affirme: «c'est une athlète, je veux qu'elle propose, qu'elle construise un cours à son image, à elle quoi, et pas un cours que j'ai décidé, ni un cours copié sur Youtube, ça non; elle est explosive, elle est entraînante, c'est sa force.»

Lagir au travail est calibré par les types d'activités qui fluctuent selon les innovations sur le marché. Il est aussi sans cesse ajusté aux profils des pratiquants, à leurs possibilités et leurs envies. Le service proposé est composé de «conduites routinières » au sein du club de fitness qui s'ordonnent autour des capacités corporelles des PF. Le niveau des cours est adapté
Planning hebdomadaire des cours proposés aux clients d'une salle de fitness

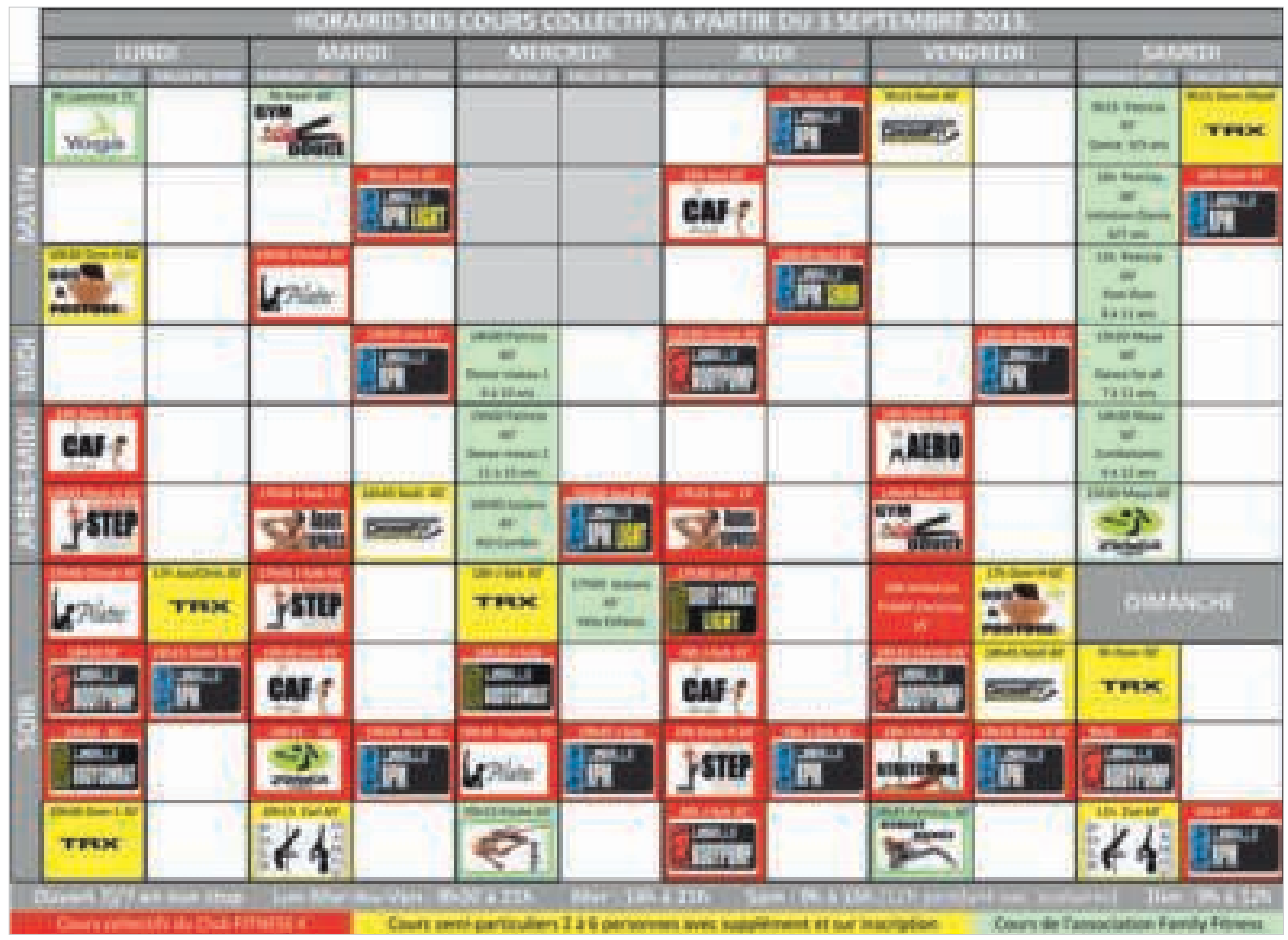




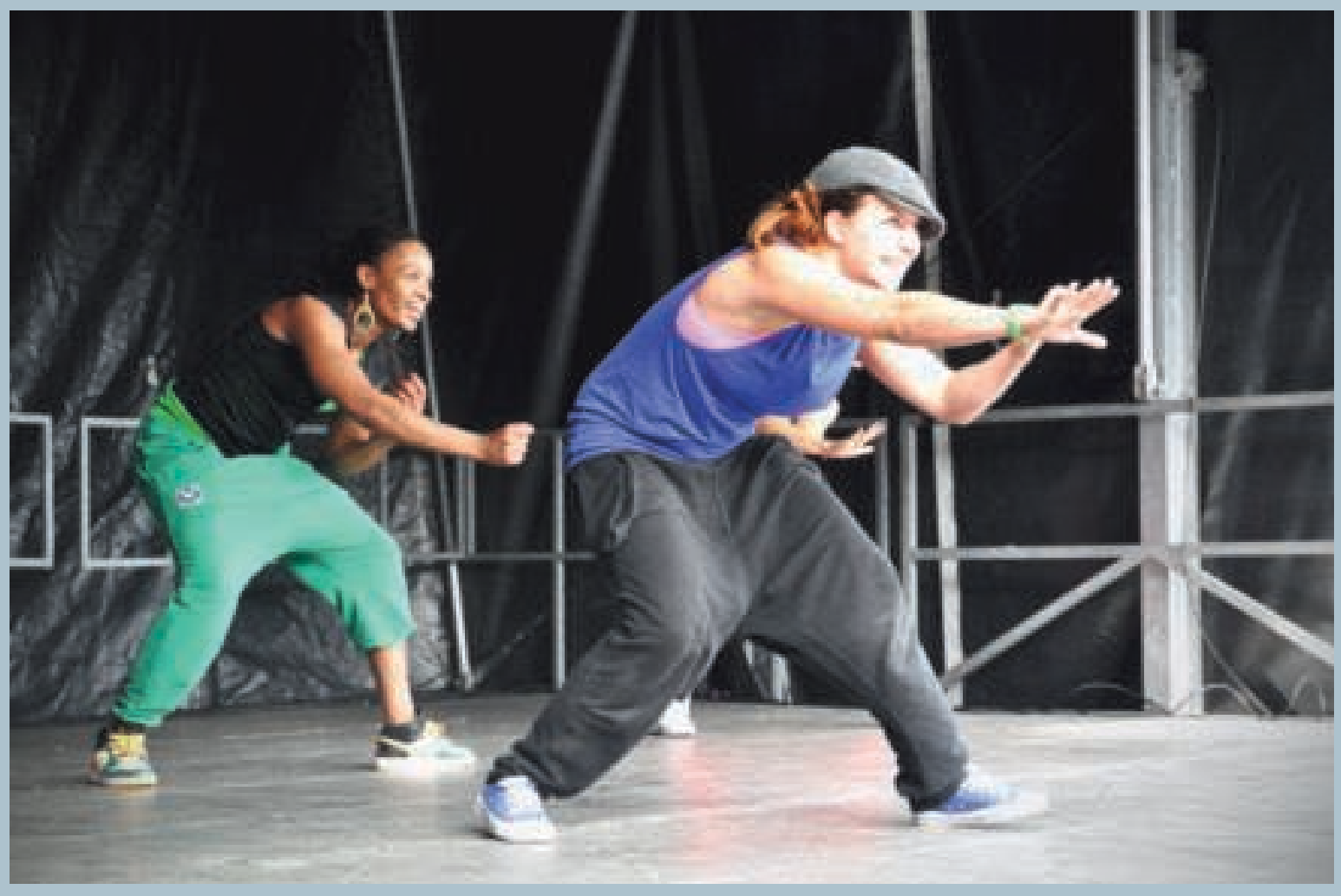

Cours collectif assuré en binôme ou team teach. 
en effet aux possibilités physiques et motrices des adhérents, à leurs desiderata du moment. Décidé par l'équipe de PF, le planning hebdomadaire des cours proposés aux clients fixe un cap et constitue le fil conducteur de leur activité routinière. La routine envisagée comme «sens et compétence impliqués dans un ajustement continu au monde», devient un moyen d'accomplissement et de disponibilité attentionnelle obligatoire dans le service au client (Breviglieri 2006). Inscrite dans une continuelle évolution et dans une dynamique individuelle d'ajustement, la routine contient des capacités dynamiques et créatives; elle atteste à la fois de l'acquisition individuelle de compétences situées et de la sensibilité permanente (en éveil) au changement (Schwint 2005). «Lorsqu'on prend en charge un pratiquant, quand on fait du coaching sur un cycle de plusieurs séances, on est amené parfois à revoir notre programme; à un moment donné, il faut adapter notre contenu, nos exercices, à la volonté du pratiquant qui revoit ses ambitions, qui est plus ou moins ou pas du tout motivé; ce n'est pas toujours cohérent d'après ce qu’on avait défini au départ, mais c'est ça le coaching, l'adaptation..., il faut composer...» (L., PF salarié et coach sportif indépendant, 33 ans).

Les travaux de Conein (1997) ont montré comment la routine ne pouvait être appréhendée de façon restreinte comme une action réglée ni par l'habitude pratique, l'instruction technique, ni par la contrainte organisationnelle. Quand ils expriment leur agacement à l'égard des formations professionnelles «trop orientées, axées sur la compétence technique des stagiaires», les employeurs-recruteurs des salles de fitness témoignent de leur préoccupation à déceler les signes dans l'attitude qui attestent d'une disposition à agir avec et en fonction des propriétés corporelles des collaborateurs, tout comme une disposition à réagir en direct aux attentes et demandes des usagers en matière d'efforts et d'exercices corporels. Le vrai travail dépend indubitablement de cet écart entre le travail prescrit - attendu officiellement du PF et formalisé dans les référentiels d'activité - et le travail réel - ce que produit et pense produire le PF. Cet écart est en réalité défini par les destinataires du travail du PF, les usagers qui, par leurs réactions, parfois convenues, parfois imprévisibles, en appellent à cette compétence professionnelle, cette ressource multidimensionnelle - psychologique, physique, technique, comportementale - qui va permettre de combler cet écart. Un usager en échec dans la réalisation d'un exercice, peut imputer ce résultat au PF, au déficit de consignes claires, compréhensibles, à l'absence d'un environnement suffisamment sécurisé qui va l'inhiber, le freiner dans l'exécution fluide de ses mouvements. Il est du devoir du PF d'apporter une réponse satisfaisante au pratiquant en acceptant sa critique. Il doit lui apporter une réponse verbale - de nouvelles consignes immédiatement compréhensibles et transmises à chaque séquence de l'exercice - et motrice qui va l'engager moralement, physiquement et techniquement pour tendre vers la réussite du client. Il devra par exemple assurer une parade, une présence physique sécurisante, ajuster le placement corporel, la bonne posture du pratiquant, et le lui signifier pour l'encourager à poursuivre par la confiance. Le cas échéant, le PF aura l'obligation de se mettre en scène et montrer au client la réalisation séquencée de l'exercice, attestant par-là de sa disposition à le servir, sans compter son temps et ses efforts.

Ainsi, tout semble indiquer que les savoir-faire requis consistent plus dans les tâches effectives et moins dans les tâches prescrites. En ce sens la routine, entendue comme condition de stabilité de l'action collective, offre des processus d'innovation et de métamorphose des collectifs (Joseph 2004) qui dépassent le cadre des prescriptions de l'organisation (Clot 2006). L'agir corporel au travail se définit dans l'ajustement physique à autrui, dans la coordination motrice avec autrui. Le PF est à même de gérer la dispersion attentionnelle des adhérents, leur hétérogénéité technique et physique, en imaginant dans l'instant 
présent des exercices réalisables et adaptés. Dans des environnements de travail complexes, fondés sur l'interdépendance des salariés, les automatismes - décisifs - impliquent une distance aux tâches répétitives, autrement dit une relâche attentionnelle et des intentions mutuelles de coopérer (Reynaud 1998). «C'est important de voir des profs qui forment une équipe, s'entendre, s'aider, qui sont complices pendant les cours, mener le cours pendant que l'autre suit, se relâche et récupère dans les cours de team teach par exemple; et en dehors les PF se fréquentent; les clients aiment le savoir, ils y sont sensibles [...] j'arrange les emplois du temps des profs en fonction de leurs engagements privés, comme ils sont tous pris par ailleurs, je connais leurs souhaits et je colmate les brèches. » (M., $\mathrm{PF}$ «manager» responsable du recrutement des PF, 40 ans). Tout PF est alors en capacité d'intervenir «sur le champ » pour parer à une situation imprévisible comme lorsque l'un de ses collaborateurs se blesse ou n'est pas en mesure d'assurer le cours.

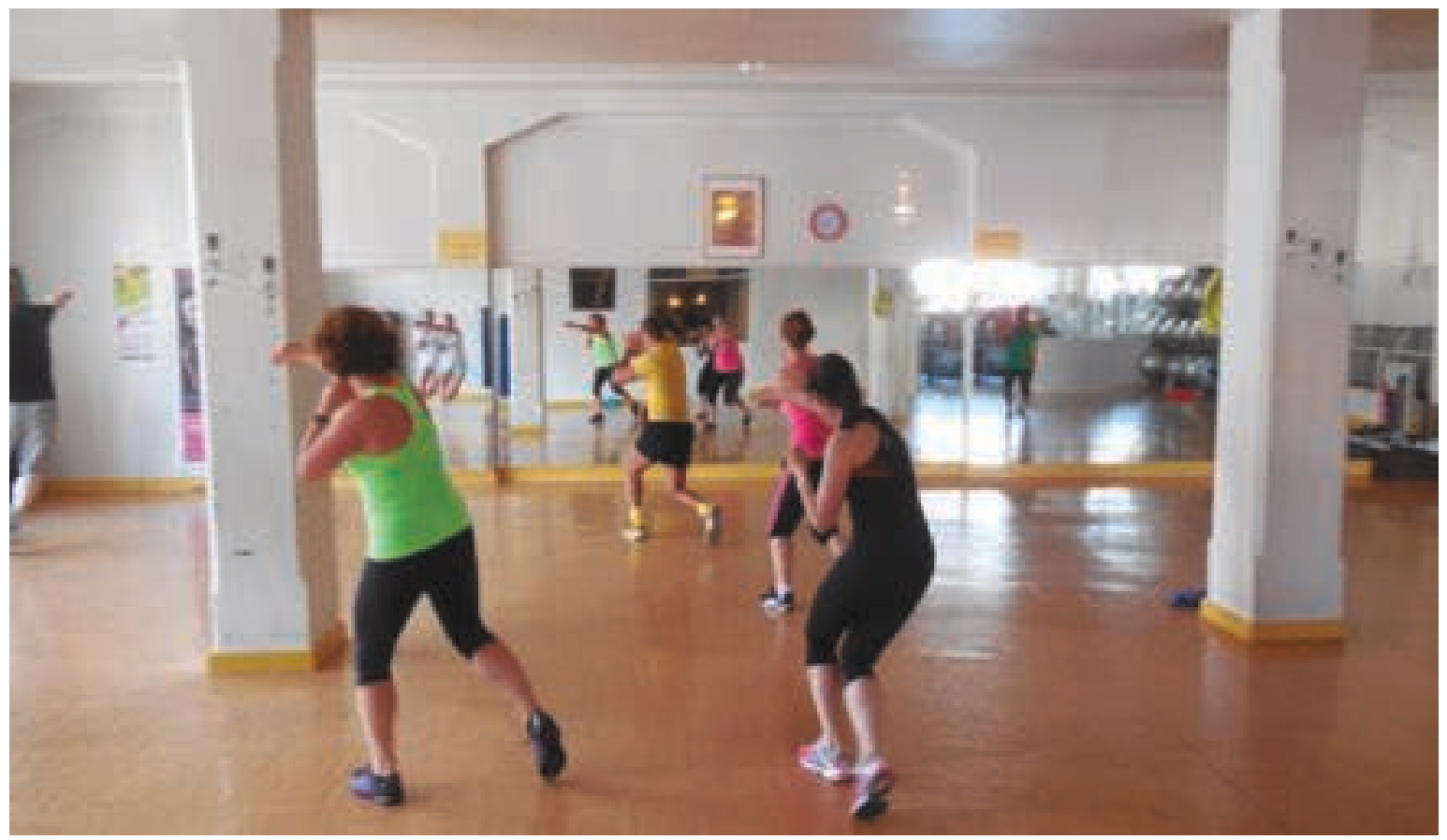

Cours collectif dans une salle bordée de miroirs

\section{S'approprier le milieu et le modifier}

L'action du professionnel dans la salle peut être qualifiée par le prisme de la rythmicité; cette insertion affective dans l'existence qui s'opère à l'aune du rapport à l'espace et aux autres significatifs (clients et employeur) des PF. La maîtrise temporelle et spatiale des PF, permise par une inscription sensorielle visible et perceptible par les personnes présentes dans les salles, contribue à la séduction des mouvements individuels, interindividuels et collectifs, à une esthétique générale. Elle exige aussi une accommodation aux codes et rituels légitimes du lieu. 


\section{Comment personnaliser léchange par l'être, le paraitre, les gestes et le mouvement}

La différenciation sociale et technique des espaces révèle un rapport à autrui différencié selon les zones d'activités. À l'impersonnalité qui semble prédominer pendant les cours collectifs regroupant plusieurs dizaines d'usagers répond une personnalisation des échanges en face à face sur le plateau de musculation. Les manières d'occuper les lieux, de communiquer avec les clients, de faire «corps» avec l'ensemble des collaborateurs pendant les temps «morts» ou d'inactivité physique, puis de se comporter en situation d'enseignement, participent de l'appropriation continue du milieu. La pression morale autour de l'esthétisme corporel se traduit par l'adoption de tenues vestimentaires correspondant aux codes idoines de chaque espace de pratique. Tout comme «être beau », soigner la présentation de soi, son image, c'est un signe de l'importance attribuée au groupe (Maisonneuve \& Bruchon-Schweitzer 1981); l'esthétisme corporel est une quête majoritairement partagée dans ces espaces dédiés à la mise en valeur et en scène des corps. Quand le plateau musculation, placé en marge des voies de passages principales de la salle, est à l'abri des regards, il est de bon aloi de porter un débardeur échancré laissant dénuder les épaules et entrevoir les muscles asséchés. Au contraire, des gérants de salle interdisent explicitement le port de débardeur échancré quand la zone de musculation est à la portée des regards, de peur que les usagers non-adeptes des exercices de musculation et peu enclins à obtenir des profits corporels visibles, se sentent discriminés face aux musculatures ostentatoires.

Les règles en vigueur dans les clubs de fitness veulent que - dans la mesure du possible, selon la taille de la structure et le nombre de clients - tout PF appelle chaque client par son prénom. Cette marque d'attention précoce dans l'interaction, qui peut paraître relever d'une superficialité démagogique, introduit une intention de personnalisation de l'échange désirant rompre avec le caractère conventionnel et impersonnel classiquement observable pour d'autres types de consommations (au supermarché par exemple). L'individualisation amicale originelle est amenée à être prolongée dans le temps, au cours de l'activité fitness; si ce n'est pas le cas, le professionnel risque d'être déconsidéré par le désaveu de cette inattention, faute de ne pas avoir honoré ses engagements. Situé au centre de l'activité économique de l'entreprise et de l'activité de travail du PF, le client et ses capacités physiques et cognitives sont l'objet d'une prise en compte minutieuse. À chaque cours collectif, le PF a obligation de prévoir des chorégraphies incluant des niveaux de difficulté optionnels variables adaptés à l'hétérogénéité du groupe de pratiquants, en vue de satisfaire leurs attentes à la fois en termes d'apprentissage, de progression et de plaisir.

Lactivité de travail du PF est assimilable à une suite de rituels. Avant d'entrer dans l'arène, c'est-à-dire dans la salle où il va dispenser son cours, il doit déambuler dans les diverses zones de pratique du club pour attester de sa présence aux yeux des clients et éventuellement en attirer quelques-uns vers son cours par l'usage contrôlé de quelques interjections amicales: "Philippe, le Bodypump commence dans dix minutes, tu me fuis ou quoi, t'as un coup de fatigue, on dirait que tu te caches...»-propos d'un PF qui taquine un des clients habitués pour le stimuler à rejoindre son cours.

Le PF fait la démonstration qu'il domine provisoirement son territoire d'expression corporelle dès qu'il accueille ses clients dans la salle d'évolution. Les échanges courtois qui précèdent le cours ont ceci de particulier qu'ils créent une dépendance, un moment intimiste où certains clients peuvent se laisser aller à leurs préoccupations personnelles. 
Si ce petit quart d'heure ne permet pas à l'instructeur de s'appesantir sur les histoires de vie de ses clients, celui-ci adopte pour autant une attitude bienveillante et compatissante tout en indiquant par son comportement son obligation à se préparer. Tout l'art commence ici: quand son inclination à rendre le client indivisible et unique, à se rendre désirable et indispensable, contribue à former l'unité d'un collectif toujours hétérogène.

\section{Ce que la technicité et le corps font au lien}

Pour convertir ses pratiquants en indéfectibles supporteurs, le PF a intérêt à préparer la réciprocité des échanges: se mettre à disposition de ses clients, prendre le temps de les écouter et d'enregistrer leurs requêtes, réunir les conditions matérielles pour favoriser la réussite du cours - rassembler et par conséquent vérifier la disponibilité des petits matériels indispensables à la réalisation des exercices corporels et des mouvements, tels que des élastiques, des ballons, des haltères à charge modérée.

Au-delà de la finalité recherchée par le biais de la tenue vestimentaire valorisant sa plastique - témoin d'une hygiène de vie irréprochable et de son ascétisme corporel ne laissant pas de place à la surcharge pondérale -, le PF transmet à son public des repères visibles pour la juste réalisation des mouvements. La technicité à l'œuvre dans les déplacements du PF est d'autant plus visible lorsque les vêtements dessinent les membres corporels et rendent limpides les phases de contraction ou de relâchement musculaire.

La gestion de l'espace et du temps, par l'entremise des mouvements réalisés par le groupe de pratiquants, participe de l'esthétique du lieu. Et la présentation de soi adaptée du PF signe ainsi sa capacité à rassembler et à fédérer l'ensemble des pratiquants ou plus exactement « d'emmener avec soi l'ensemble du groupe». Par son corps en mouvement, sa communication verbale, son toucher et ses regards, le professionnel doit constamment ouvrer à l'équilibre moteur du collectif. Les miroirs qui bordent les salles de cours prédisposent aux interactions visuelles et corporelles à distance et renforcent la préoccupation partagée d'une action et d'une unité collectives.

Ne laisser personne sur le bord du chemin, éviter que certains - les « grandes gueules toujours sûres d'elles» - «écrasent» les plus discrets, les plus introvertis. Le professeur assure alors des «opérations de dégagement qui déresponsabilisent les pratiquants dont les gestes sont inadaptés, mal exécutés, mal maîtrisés, dont les propos sont maladroits, bref, des manières d'être, de dire et de faire incongrues» (Pichot \& Wipf 2011: 140). Mais l'enjeu de son comportement consiste à maintenir l'attention du plus grand nombre: cette concentration obtenue par la compréhension d'un langage verbal et corporel simple, accessible, signifiant et générateur de mouvements correctement réalisés. Ainsi la réussite du mouvement conduit à préserver l'individualité, le «soi » de chacun, indépendamment des codes oppressants du milieu qui tentent d'infliger une auto-discrimination des pratiquants. Le PF a justement pour mission de rompre avec les injonctions ambiantes, les codes indigènes parfois accablants, susceptibles de stigmatiser les adhérents: «tu vois, moi je fais cours avec un pantalon de survêt en coton, le sportif de base quoi, celui que tu peux voir sur le bord des terrains de foot le dimanche; ça dénote dans le milieu, mais je m'en fous, je suis loin des tenues moulantes, mais je crois que c'est ça qui plaît parce qu'il y a un public qui me ressemble, qui ne s'embête pas des qu'en dira-t-on... » (C., 39 ans, PF, 10 ans d'ancienneté dans le métier). 


\section{Quand voir, percevoir et sentir, cest agir, faire faire et unir}

Le PF n'est jamais un être achevé, complètement constitué. Il peut ouvrir un espace de pratique de soi « où rénover ses capacités, travailler ses contradictions, apprendre à s'orienter autrement dans ses propres possibles, à naviguer d'une disposition à une autre, et, si l'on peut dire, à se redisposer » (Bidet \& Macé 2011 : 273). L'ouverture d'un horizon d'anticipation est inhérente à la question de l'individuation.

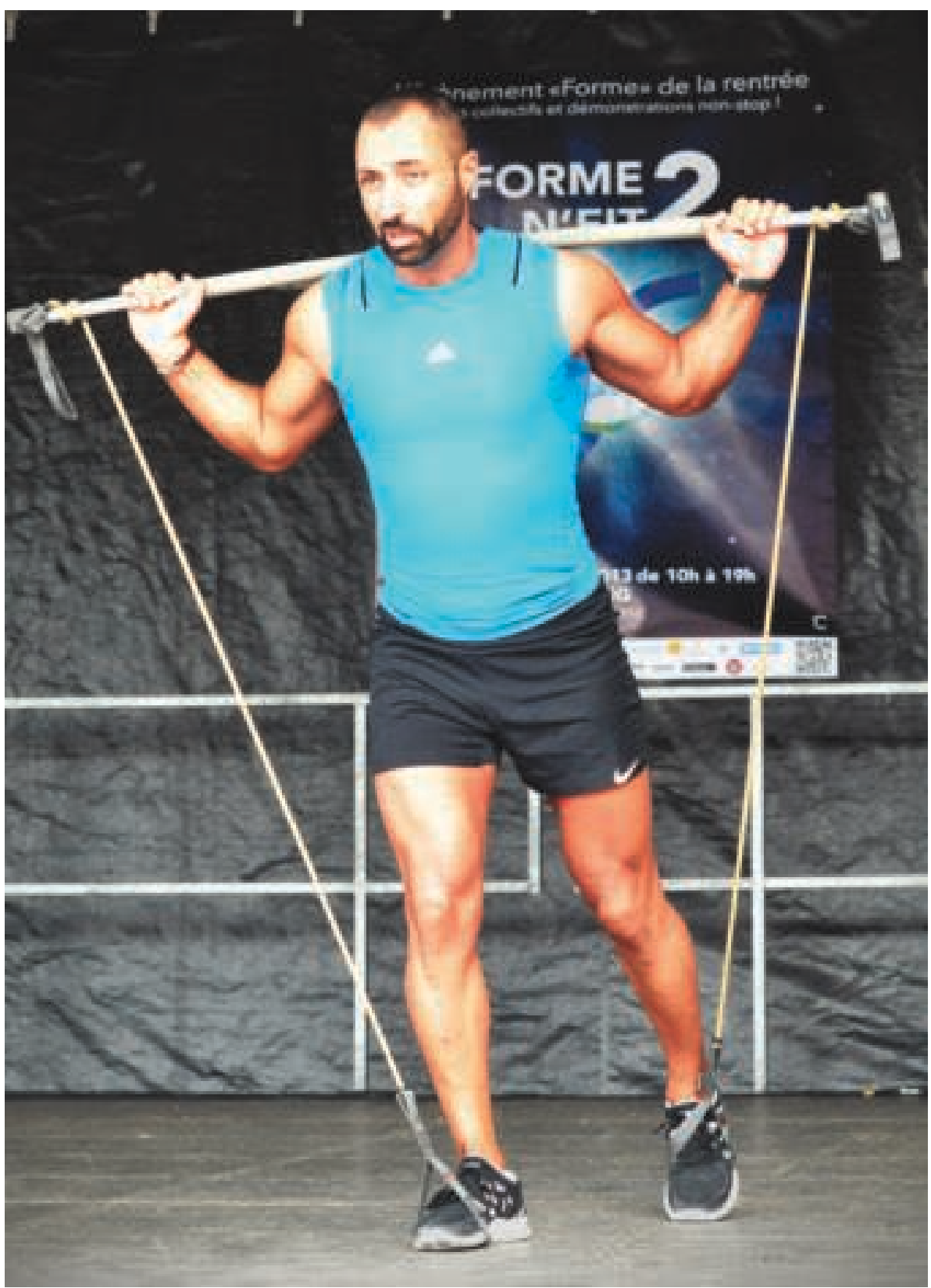

PF portant une tenue vestimentaire moulante spécifique, visant à mettre en relief sa musculature asséchée 
L'examen des engagements en tension, ou pour le moins prenant des directions obliques de S. - PF depuis une quinzaine d'années - est instructif. L'évolution de ses interventions dans la même salle depuis plus de dix ans est révélatrice de l'adaptation continue au milieu: «Au départ, quand tu fais du suivi en plateau de musculation, c'est intéressant de t'adapter à l'autre, c'est la passion du coaching. C'est-à-dire c'est capter comment tu peux jouer avec ton corps, comment tu peux prendre du plaisir avec ton corps; on peut aimer ça, ça fait partie du bien-être. Et la musculation peut aider à corriger certaines choses [...]. C'est pour ça qu'on peut gagner de la confiance en soi par la musculation, on peut se défaire de certains complexes de son corps; je pense que les cours collectifs ne suffisent pas par rapport à un objectif esthétique et les gens viennent beaucoup pour ça. Dans les cours collectifs, l'épanouissement passe par l'échange, plus que par la technique ou le dépassement de soi. En fait tu donnes de l'énergie, et tu reçois de l'énergie en retour. Moi je donne des cours collectifs le matin, j'ai donc des petits groupeset le challenge, c'est d'en avoir toujours plus [des adhérents] car le soir de toute façon ils sont là, tu n'as pas besoin d'aller les chercher, il n'y a pas de challenge; c'est intéressant les petits groupes car c'est plus facile pour corriger, et corriger, c'est se rendre utile [...]. Le soir, c'est autre chose, le show, les cris. J'ai un cours de Bodystep le lundi soir: je donne beaucoup, je montre les options, les versions plus calmes, c'est pour montrer aux gens qu'il y a d'autres manières de bouger, de faire les exercices.»

Cet extrait d'entretien condense diverses facettes de ce métier, valorisé par l'utilité sociale inhérente à la logique de service: "prendre soin de son client». Au centre, l'éducation corporelle et l'échange social prennent des formes différentes selon les activités et les moments, tour à tour intimiste, en face-à-face; impersonnel, dans les groupes de pratiquants élargis. Dans tous les cas, l'accomplissement de soi visé par le PF repose sur la confiance établie, l'interaction verbale ou physique, sources de plaisir. De ce fait, le contact effectif entre le PF et ses adhérents structure un environnement durable (Quéré 2006). Devenue responsable du planning des PF, S. doit accorder toutes les compétences réunies pour former une équipe pédagogique tendant vers un même objectif: satisfaire le plus grand nombre d'adhérents ${ }^{5}$. «Mon job, c'est aussi de faire en sorte que les profs puissent exprimer tout leur potentiel, donc à travers les cours qu'ils préfèrent, dans lesquels ils se sentent le mieux, c'est normal... [...] À moi aussi de les protéger, physiquement, de gérer les temps forts et les temps creux, car il y a toujours quelque chose à faire en suivi sur le plateau de musculation [...] à moi aussi de gérer les baisses de régime, car le job est difficile physiquement, usant mentalement. »

Sous un autre angle de vue, B. nous éclaire sur les différents engagements spécifiques au métier: «En 2003, j'étais aussi sur le plateau de musculation pour le suivi, la surveillance. Ce qui était gratifiant, c'était le suivi du programme par le client et le résultat obtenu [...]. Maintenant je donne uniquement des cours collectifs, type Sh'bam, c'est un cours dansé, de Bodybalance, c'est un cours de 30 minutes, renforcement abdominal, des épaules et des fessiers, de Bodypump, de Bodycombat [...] et aujourd'hui ma mission principale a évolué vers l'administratif et l'aménagement intérieur de la salle, la décoration, c'est ce que je préfère maintenant. » Reconnu à la fois pour sa créativité en cours de danse, pour ses idées originales au service de l'aménagement des espaces de travail, et aussi pour sa rigueur administrative et comptable ${ }^{6}$, B. séduit collaborateurs et pratiquants pour ses potentiels hétérogènes: «B. il est énorme, c'est la classe, physiquement, humainement, et sans le faire voir, et il a quand même 40 ans passés ».

Trouvant son origine dans un collectif diffus d'humains, l'acte individuel participe de l'autoconstitution du sujet. Lors d'un cours dispensé dans un espace restreint, la masse 
de pratiquants fonctionne comme paravent, elle permet à tout un chacun de rester anonyme. L'insertion du pratiquant dans le collectif favorise l'engagement individuel spontané, sans calcul. Ses prises de repères sont à rechercher dans ses conduites antérieures et le mouvement individuel se forge dans le mimétisme et résulte d'itérations ajustées à la situation présente, selon le rythme des exercices et les espaces proximaux investis. L'individu constitue son milieu et se structure en même temps qu'il agit. En ce sens, l'individuation peut être assimilée à la coproduction entre l'individu - le pré-individuel

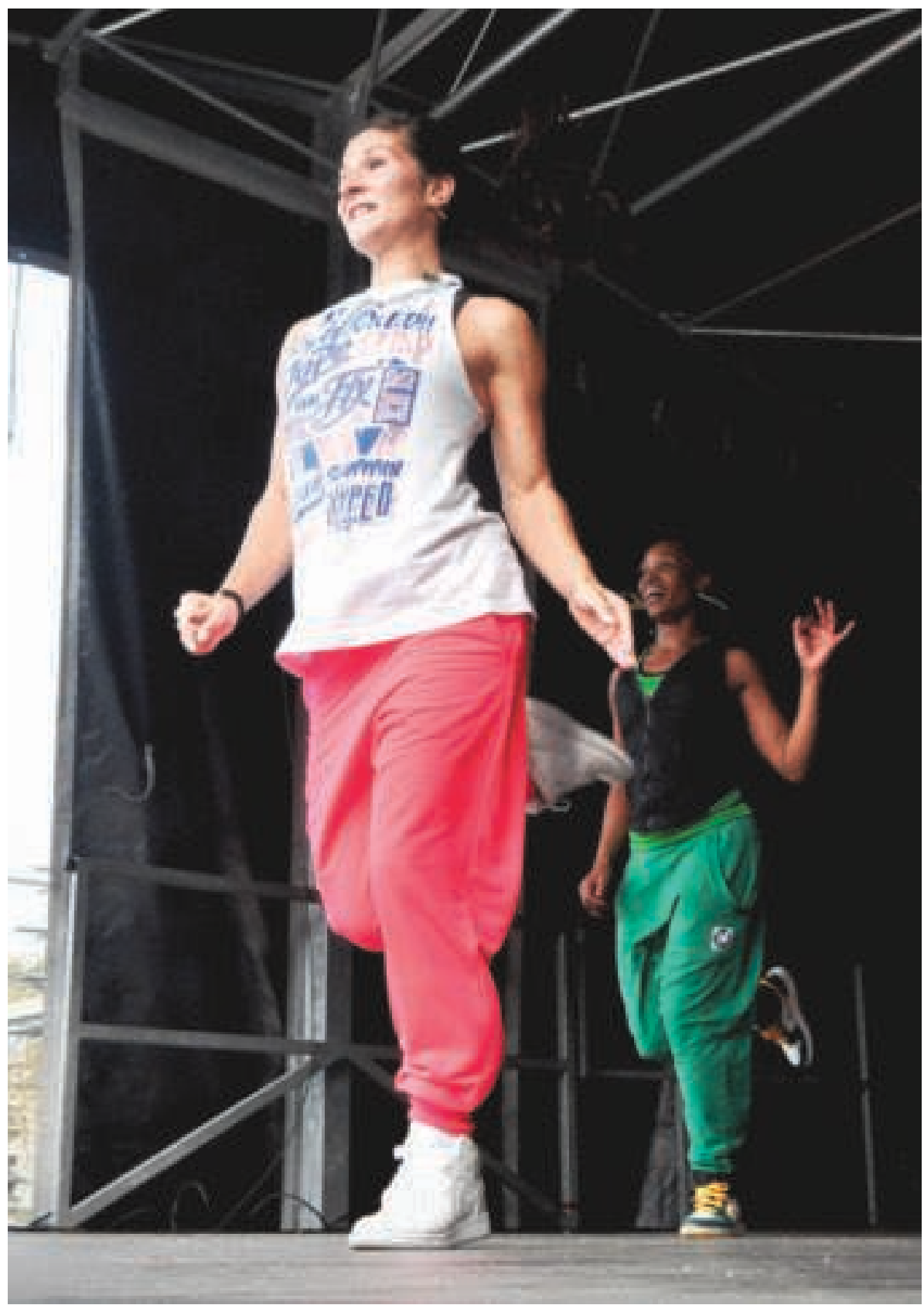

PF portant une tenue de sport en coton anodine, non spécifique à l'univers du fitness 
même - et le milieu. Le passage du PF dans les diverses salles du club, avant le début de son cours, est le signe annonciateur du rassemblement des pratiquants intéressés. Leur relative impatience et leur excitation corporelle trouvent en la personne du PF un point d'attache et la possibilité d'une communication non verbale. L'échange social et l'émancipation expriment l'individuation. L'insertion naturelle et réussie de l'individu dans «son» milieu doit être « comprise comme un régime réciproque d'échanges d'informations et de causalité dans un système qui s'individue » (Simondon 1989: 210). Selon une prédisposition au collectif, l'individu aspire à se structurer et une part d'indétermination pousse à l'incorporation de ce dernier dans le collectif. La posture d'accompagnant, implicitement réclamé au PF, traduit l'engagement affectif dans les relations au travail, posture éloignée et affranchie du mode injonctif qui consiste à motiver par le «il faut ».

Les rapports d'identification interindividuels se forment à travers les activités motrices réciproques du PF et de ses adhérents, leurs échanges mutuels cristallisés par des propriétés communes - le partage de sensations et d'émotions, des difficultés d'exécution ou des instants de réussite qui rendent solidaires et qui rassemblent -, des attitudes, un langage, une tenue vestimentaire. Ces éléments de situation, à forte charge émotionnelle - «se voir», «se comprendre », «sentir ce que l'autre ressent»-font émerger un collectif où la séduction interindividuelle opère. Le collectif naît d'une rythmicité qui produit une unité, qui entraîne dans un même mouvement et qui s'appuie sur des valeurs morales: l'investissement de soi (du PF) est une condition et, le résultat de l'accomplissement de soi d'autrui (le client) est le ferment de la reconnaissance sociale au travail pour soi-même. Ce que signifient «se sentir bien », «être dans mon élément », «je suis bien dans ce cours, dans cette mission »- des propos banals répétés -, nous donnent à théoriser un sentiment de familiarité dominant et partagé chez les PF qui s'éprouve dans l'ordre préréflexif du mouvement (Bidet 2007 b) et qui traduit «la conscience d'un accompagnement moteur bien réglé, d’une réaction motrice organisée» (Merleau-Ponty 1993: 171).

\section{$\&$}

L'expérience fait sens concomitamment à l'émancipation (Bidet \& Macé 2011); c'est-àdire à l'appropriation maîtrisée, mais jamais absolue, d'un cadre d'action normatif et plastique, en même temps qu'elle libère des exercices répétés et standardisés. Les rythmes et les styles se déploient à l'échelle inter et intra-individuelle, dans un double ajustement, externe avec les clients-pratiquants et interne selon une auto-perception de soi par les autres et soi-même. Aussi s'accomplir au travail doit être compris comme une insertion dans l'existence émotionnellement vécue, par le lien entre corporéité et socialité, entre affect et symbole. Le PF vit par le milieu, l'environnement qu'il contribue à façonner et les relations qu'il a sues et pu y nouer. En d'autres termes, les groupements collectifs, ici les microcollectifs, se constituent à partir de représentations et d'actions elles-mêmes fondées sur des émotions, des sensations corporelles éprouvées.

En ce sens, l'activité éprouvée sur le mode de l'engagement dans son milieu et de la distanciation par rapport à l'instantanéité des relations s'apparente à une rythmicité composée de phases d'extrêmes concentrations - la correction en direct des postures mal ajustées des clients - et de relâches attentionnelles (Bidet 2011 b) - « chambrer » amicalement les clients aguerris sur des mouvements mal exécutés ou sur des prises d'initiatives 
risquées qui traduisent une surestimation de leur propre potentiel moteur. Le travail, compris comme une expérience vécue mêlant à la fois le présentisme et la réflexivité, permet de saisir de nouvelles formes de socialité et d'action réciproque, notamment des déphasages temporels et des rythmicités opposés entre professionnels.

Lactivité de travail ne résulte pas d'une planification, elle favorise au contraire une unification de l'expérience qui repose non pas sur des formats ou des dispositifs établis mais sur des rythmes. La perspective sociologique pragmatiste et anthropologique du travail inspire une approche de la multi-activité au travail comme «concours de distribution et d'unification» et comme expérience (Bidet 2011b). La transformation continue des activités elles-mêmes se traduit par l'enchâssement des engagements hétérogènes: être performant pendant le cours, être disponible mentalement avant et après le cours pour discuter "de la pluie et du beau temps», "écouter les états d'âme des clients", des choses ordinaires de la vie... L'émancipation du PF au travail présente un caractère construit: elle est consubstantielle du travail du temps de l'activité qui donne à voir les deux traits de ce qu'on appelle le «vrai boulot » : les repères pour agir et les formes de félicité éprouvée qui sont liés à l'histoire des relations entretenues par les individus.

Lactivité de travail considérée comme une somme d'actions infinies, jamais achevées, est enrichie par la mémoire, par la formation d'habitudes gestuelles et langagières et d'exigences corporelles ou posturales qui guident l'action et lui donnent de la valeur (Pillon 2012). Sa valeur pratique et cognitive immanente à la rythmicité permet d'affirmer que l'agir au travail est inséparable d'une connaissance fine des attentes et des potentialités des destinataires du service et de réactions adéquates à celles-ci. En cela, elle invite plus que jamais les acteurs en coprésence à l'échange actif sur des modes communicatifs variés et complémentaires et, à l'expérience de l'altérité.

\section{NOTES}

Photo d'ouverture: La prise en charge individuelle d'un pratiquant par le professeur de fitness.

Toutes les illustrations sont de l'auteur.

1. Soit être présent physiquement dans l'espace d'expression corporelle, être présent dans la structure sans être vu, sans être là, s'effacer.

2. Ces activités aquatiques, présentées et commercialisées comme ayant des vertus d'embellissement corporel, sont censées raffermir et tonifier le corps et les muscles des membres inférieurs (jambes, cuisses et fesses).

3. Il s'agit d'un enchaînement d'exercices présentés sous la forme d'ateliers dont la succession logique est déterminée par la fonction des appareils qui exigent dans leur utilisation une force musculaire, une expertise telle qu'ils fixent l'ordre chronologique de la séquence.
4. Dodier est cité par Ughetto dans son article «Introduction. Le travail comme activité et comme action. Quelques perspectives sociologiques récentes» (2004: 121-139).

5. La salle où travaille $S$. accueille près de 5000 clients et emploie 10 professeurs de fitness pour des activités sportives dites terrestres et aquatiques.

6. Âgé de 41 ans, B. est titulaire d'un bac G2 spécialisé en comptabilité. 


\section{RÉFÉRENCES}

Bidet, A. et al. 2006 Sociologie du travail et activité. Toulouse: Octarès.

Bidet, A. 2007a «Le corps, le rythme et l'esthétique sociale chez André Leroi-Gourhan», TechniquesE Culture 48-49: 15-38.

Bidet, A. 2007b «Le travail entre corps et technique: du labor à l'agir créatif », Communications 81 : 215-223.

Bidet, A. 2011 la «Le lien social» in A. Bidet L'engagement dans le travail. Qu'est-ce que le vrai boulot? Paris: PUF.

Bidet, A. 201 lb «La multi-activité, ou le travail est-il encore une expérience? », Communications, 89: 9-26.

Bidet, A. \& M. Macé 2011 «S'individuer, s'émanciper, risquer un style (autour de Simondon)», Revue du Mauss 38: 269-284.

Breviglieri, M. 2006 «Perceptions sociologiques du problème de la routine» in A. Bidet et al. dir. Sociologie du travail et activité. Toulouse: Octarès: 131-141.

Clot, Y. 2006 [1995] La fonction psychologique du travail. Paris: PUF.

Conein, B. 1997 «Laction avec les objets. Un autre visage de l'action située? » in B. Conein \& L. Thévenot dir. Cognition et information en société. Paris : Éditions de l'EHESS : 25-46.

Datchary, C. 2008 «Gérer la dispersion: un travail collectif», Sociologie du travail (50) 3: 396-416.

Dodier, N. 1995 Les Hommes et les machines. La conscience collective dans les sociétés technicisées. Paris: Métailié.

Ernst, A. \& C. Pigeassou 2005 «"Être seuls ensemble": une figure moderne du lien social dans les centres de remise en forme», Movement $\mathcal{E}$ Sport Sciences 56(3): 5-74.

Goffman, E. 1973 La Présentation de soi. Paris: Éditions de Minuit.

Gold, R. 1958 «Roles in Sociological Field Observations», Social Forces 36 (3) : 217-233.

Haissat, S. \& Y. Travaillot 2012, «La pratique dans les centres de remise en forme: des interactions normalisatrices entre les pratiquantes », Movement E Sport Sciences 76 (2): 33-37.

Joseph, I. 1998 Erving Goffman et la microsociologie. Paris: PUF.

Joseph, I. 2004 Météor. Les métamorphoses du métro. Paris: Economica.

Leroi-Gourhan, A. 1964 Le geste et la parole, I. Technique et langage. Paris: Albin Michel.

Leroi-Gourhan, A. 1965 Le geste et la parole, II. La Mémoire et les rythmes. Paris: Albin Michel.

Maisonneuve, J. \& M. Bruchon-Schweitzer 1981 Modèles du corps et psychologie esthétique. Paris: PUF.

Merleau-Ponty, M. 1993 [1945] Phénoménologie de la perception. Paris: Gallimard.

Mischler, S. \& L. Pichot 2005 «La nécessaire diversification des politiques de salles de fitness. Les logiques d'action des entreprises de la forme en Alsace », Loisir E Société/Society E Leisure 28 (1) : 239-263.

Pichot, L. \& L. Wipf 2011 «Le professeur de fitness au travail: regard ethnographique en situation », Communications 89: 133-146.

Pillon, T. 2012 Le corps à l'ouvrage. Paris: Plon.

Pillon, T. et al. 2011 «Travailler», Communications 89.

Quéré, L. 2006 «L'environnement comme partenaire» in J.-M. Barbier \& M. Durand dir. Sujets, Activités, Environnements. Approches transverses. Paris: PUF.

Schwint, D. 2005 « La routine dans le travail de l'artisan», Ethnologie française 3: 503-512.

Reynaud, B. 1998 «Les propriétés des routines: outils pragmatiques de décision et modes de coordination collective », Sociologie du travail 4 : 465-477.

Sennett, R. 2000 [1998] Le travail sans qualités. Les conséquences humaines de la flexibilité. Paris: Albin Michel. Simondon, G. 1989 Lindividuation psychique et collective. Paris: Aubier Montaigne.

Ughetto, P. 2004 «Introduction. Le travail comme activité et comme action. Quelques perspectives sociologiques récentes», Revue de l’Ires 44: 121-139.

\section{POUR CITER CET ARTICLE}

Pichot, L. 2017 «L'agir au travail du professeur de fitness. Le lien social rythmé par le corps et la technique » in G. Bartholeyns \& F. Joulian, Le corps instrument, Techniques\&Culture 62 : 252-271. 


\section{RÉSUMÉ}

L'agir au travail du professeur de fitness. Le lien social rythmé par le corps et la technique.

Comment un travailleur tel que le professeur de fitness, éducateur sportif spécifique, s'accommode de l'hétérogénéité matérielle et humaine qui caractérise son lieu de travail? C'est en répondant à cette question que l'article décrypte les manières dont les expressions corporelles et humaines propres à l'univers du fitness reposent sur une valeur technique et font non moins référence à des valeurs morales. Au sein d'un ensemble humain fort différencié, situé dans un cadre spatio-temporel non moins hétéroclite, la solidarité technique (Dodier 1995) autour d'un collectif co-construit marque l'expérience de travail du professeur de fitness qui y construit son identité. Celle-ci est marquée par la rythmicité (Leroi-Gourhan 1965) entendue comme une insertion affective, sentimentale et corporelle située au cœur d'un milieu, fait de liens matériels et humains. L'examen de «l'activité en train de se faire», solidaire d'une approche anthropologique du travail (Bidet 2011), s'applique ici à débusquer les ressources adaptatives des professeurs de fitness ajustées continûment aux incertitudes qui rythment les phases successives au travail. Ces ressources, acquises et travaillées, sont le fruit d'arrangements plus improvisés que planifiés, même s'ils sont maîtrisés, et reposent en grande partie sur des engagements sensibles, émotionnels et moraux. Elles démontrent que l'activité de travail signifiante du professeur de fitness doit être explorée en tant que processus relationnel, comme sa relation engagée dans et par l'environnement matériel et humain.

\section{ABSTRACT}

The fitness instructor's action at work. Social bond through body and technique

How a worker such as fitness instructor, specific sport instructor, accommodates to physical and human heterogeneity in his workplace? It is in answering at this question that this article decrypts the ways in which human body and unique expressions to the world of fitness are based on technical merit and no less refered to moral values. In a very differentiated human group, located in an equally diverse spatio-temporal framework, technical solidarity (Dodier 1995) about a group co-built brand experience working, the fitness instructor builds his identity. It is marked by the rhythmicity (Leroi-Gourhan 1965) understood as an emotional integration, sensitive and bodily situated in the heart of a circle made of material and human relationships. The examination of « the activity in way to be done» interdependant to an anthropological approach to work (Bidet 2011), applies here to uncover adaptive resource fitness instructor adjusts permanently to uncertainties that mark the successive phases of his work. These resources acquired and worked out, are the result of more improvised arrangements than planned, even if they are controlled, and largely based on sensitive commitments, emotional and moral as well. They demonstrate that the activity of meaningful work of fitness instructor should be explored as a relational process, as well his relation engaged in and through human and materiel environment.

\section{MOTS CLÉS}

Professeur de fitness, agir au travail, solidarité technique, collectif de travail, expérience, rythmicité

\section{KEYWORDS}

Fitness instructor, acting in working, technical solidarity, collective work, experience, rhythmicity 